\title{
The Equations of Lorentz Transformation
}

\author{
Csizmadia Jozsef \\ Inovator Plus Ltd. Kanjiza, Kanjiza, Serbia \\ Email: savap123456@hotmail.com
}

Received 29 March 2016; accepted 24 May 2016; published 27 May 2016

Copyright (C) 2016 by author and Scientific Research Publishing Inc.

This work is licensed under the Creative Commons Attribution International License (CC BY).

http://creativecommons.org/licenses/by/4.0/

(c) (i) Open Access

\section{Abstract}

This work consists of two parts. The first part: The Lorentz transformation has two derivations. One of the derivations can be found in the references at the end of the work in the "Appendix I" of the book marked by number one. The equations for this derivation [1]:

$x^{\prime}=\frac{x-v t}{\sqrt{1-\left(v^{2} / c^{2}\right)}} ; t^{\prime}=\frac{t-\left(v / c^{2}\right) x}{\sqrt{1-\left(v^{2} / c^{2}\right)}}$, The other derivation of the Lorentz transformation is the traditional hyperbolic equations: $\quad c t^{\prime}=c t \cosh \xi-x \sinh \xi ; \quad x^{\prime}=x \cosh \xi-c t \sinh \xi ; \quad \zeta=\operatorname{artanh}(v / c)$. For these equations we found new equations: $c t^{\prime}=c t \cosh \xi-x \sinh \xi=c t(1 / \sin \varphi)-x \operatorname{cotan} \varphi$; $x^{\prime}=x \cosh \xi-c t \sinh \xi=x(1 / \sin \varphi)-c t \operatorname{cotan} \varphi$. The second part: In the second part is the $\left(t^{\prime} / t=\tan (\varphi / 2)\right)$ equation by which we derive Minkowski's equation. It will be proved that Minkowski's equation is the integral part of the Lorentz transformation.

\section{Keywords}

\section{Lorentz Transformation, Relativity, New Equations}

\section{Introduction}

The aim of this work is to express the hyperbolic equations by trigonometric equations. I would like to point out the simplicity of the used triangles by the derivation of the equations. This work is based on three equations. In the first part of this work we work with two equations:

New equation: $(1 / \sin \varphi)=\cosh \xi$.

New equation: $\operatorname{cotan} \varphi=\sinh \xi$.

We join them by the hyperbolic equation of Lorentz transformation. The new equations give the same results as the Lorentz transformation hyperbolic forms. For the derivation of the new equations high school mathematics is used. That's the reason why the new equations are more transparent and easier to understand because they 
are simply. After this, we check several ways the correctness of the new equations. The new equations must be in accordance with Minkowsi's equation. In the second part we study the $t^{\prime}$ equations which we derived in the first part. Here we focus on the case when $x=c t . x=c t$ in the first part appearing equations is a special case. Here we introduce our third new equation:

$$
\frac{t^{\prime}}{t}=\tan (\varphi / 2)=\cosh \xi-\sinh \xi=\mathrm{e}^{-\xi}
$$

We shall proof as well that the $(\tan (\varphi / 2))$ equation and the hyperbolic equations are connected to each other. It is very important that the $\left(t^{\prime} / t\right)=(\tan (\varphi / 2))$ equation should be in accordance with Minkowsi's equation. We want to show the importance of the $\left(t^{\prime} / t\right)=(\tan (\varphi / 2))$ equation. That's the reason why the Minkowski equation is to be derived by the equation $\left(t^{\prime} / t\right)=(\tan (\varphi / 2))$, and show how Minkowski equation links to Lorentz transformation. Minkowski's equation can be seen in the "Appendix I" of the book marked by number one in the references in [1]: $\left(x^{\prime 2}-c^{2} t^{\prime 2}=x^{2}-c^{2} t^{2}\right)$.

\section{The First Part}

\subsection{First Come the New Equations}

Now come the traditional hyperbolic equations [2] [3]:

1) $c t^{\prime}=c t \cosh \xi-x \sinh \xi$.

2) $x^{\prime}=x \cosh \xi-c t \sinh \xi$.

3) $\xi=\operatorname{artanh}(\beta)$.

4) $\beta=(v / c)$.

Instead of these I found new equations, and we shall derive them. First come the new equations, and then their derivations.

5) $x^{\prime}=x \cosh \xi-c t \sinh \xi=x(1 / \sin \varphi)-c t \operatorname{cotan} \varphi$.

6) $c t^{\prime}=c t \cosh \xi-x \sinh \xi=c t(1 / \sin \varphi)-x \operatorname{cotan} \varphi$.

The aim is that in the Equations ((1) and (2)) appear in hyperbolic equations to express the trigonometric equations.

\subsection{The Triangle and the Hyperbola}

The triangle:

7) See Figure 1, triangle $O A D: \cos \varphi=(v / c)$.

8) See Figure 1, $\varphi=\arccos (v / c) \quad$ radian .

9) See Figure 1, triangle $O A D$, and Equation (7)):

$$
\sin \varphi=\frac{O A}{O D}=\frac{O A}{1}=O A=\sqrt{1-\cos ^{2} \varphi}=\sqrt{1-\left(v^{2} / c^{2}\right)}\{\text { radian }\} .
$$

The hyperbolic function was introduced into the mathematics on the analogy of Figure 1.

The hyperbola simplest form is: $x^{2}-y^{2}=1$

See Figure 2:

10) $\tanh \xi=E G=(v / c)$.

11) $\sinh \xi=F H$.

12) $\cos \xi=O F$.

13) Grey area ( $\xi)$. See Equation (3) and [3]: $\xi=\operatorname{artanh}(v / c)$.

(Note: Figure 1 and Figure 2 can be found in [4]).

14) Course Equation (10), is identical with Equation (7). See Equations ((7) and (10)):

$$
\begin{gathered}
\cos \varphi=(v / c)\{\mathrm{rad}\} \\
\tanh \xi=(v / c) \\
\text { So }: \cos \varphi=\tanh \xi=(v / c)=(v t / c t)\{\operatorname{rad}\}
\end{gathered}
$$

Figure 3 = Figure $1+$ Figure 2 . 


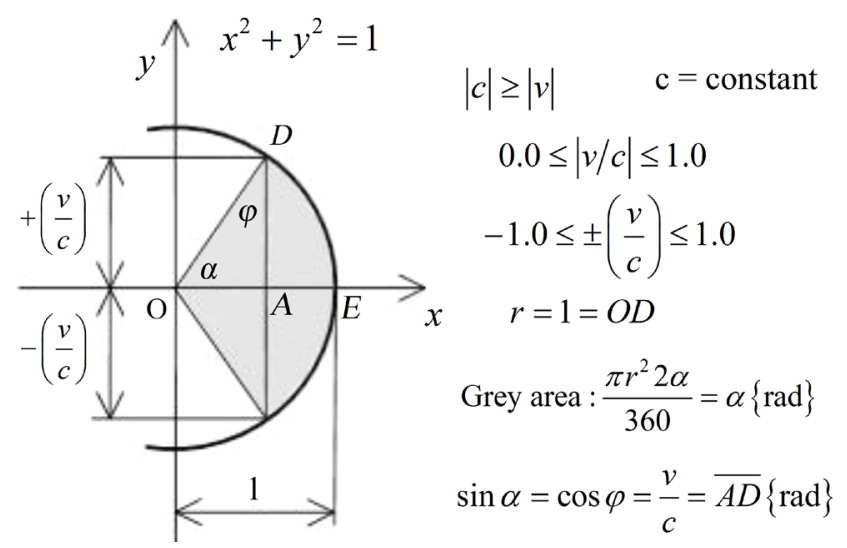

Figure 1. The $O D$ section revolves around the $O$ point. The more we reduce the " $v$ " value, the smaller will be the " $\alpha$ " angle, however, the " $\varphi$ " becomes bigger.

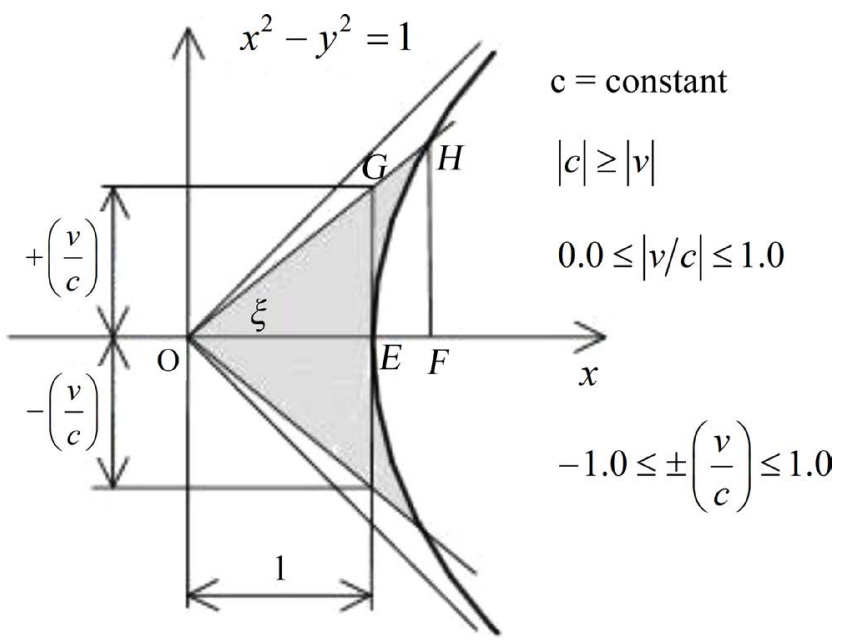

Figure 2. The $\mathrm{OH}$ section turns around the $O$ point. The $H$ point is a point of hyperbola. The more we reduce the " $v$ " value, the smaller will be the " $\xi$ " value.

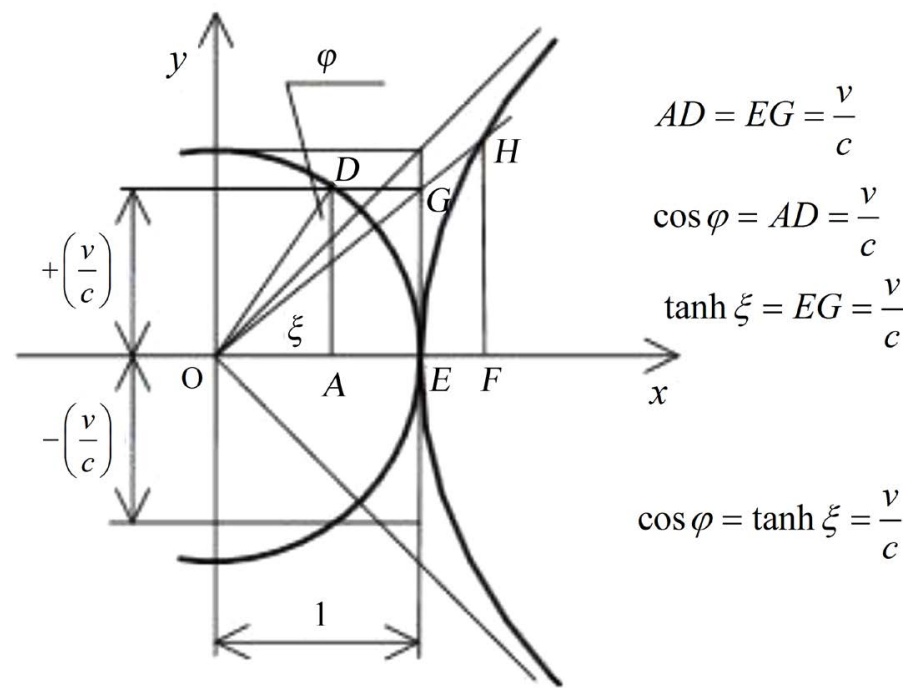

Figure 3. The $D$ point moves on the circle, the $H$ point moves on the hyperbola. The more we reduce the " $v$ " value, the bigger will be the " $\varphi$ " angle, however, the " $\xi$ ' angle becomes smaller. 
Figure 3: The point is that if we change the value of " $v$ " the $D$ and the $H$ points position changes too. From $D$ point situation depends the $H$ point situation. That is to say, each point on the circle is assigned to a point of hyperbole. The value of the " $\varphi$ " and " $\xi$ " variables defines the $v$ variable size. The value of $c$ is constant.

\subsection{The First New Equation}

We shall express the ( $\cosh \xi$ ) and ( $\sinh \xi$ ) variables in the Equations ((1) and (2)) with new equations.

First we prove that: $(1 / \sin \varphi)=\cosh \xi$. After that we prove that $\operatorname{cotan} \varphi=\sinh \xi$. Then we substitute these in the Equations ((1) and (2)).

The $\{\cos \varphi=\tanh \xi=(v / c)\}$ allows to find connection between $(1 / \sin \varphi)$ and $(\cosh \xi)$. In fact, the connection is sought between the $O A D$ triangle Figure 3 and $O E G$ triangle Figure 3 and $O F H$ triangle Figure 3.

Now follow the derivation of equation $(1 / \sin \varphi)=\cosh \xi$.

15) We substitute the Equation (14): $\{\cos \varphi=\tanh \xi=(v / c)\}$ into the Equation (9):

$$
\begin{aligned}
\sin \varphi & =\sqrt{1-\cos ^{2} \varphi}=\sqrt{1-\left(v^{2} / c^{2}\right)}=\sqrt{1-\tanh ^{2} \xi}=\sqrt{1-\frac{\sinh ^{2} \xi}{\cosh ^{2} \xi}} \\
& =\sqrt{\frac{\cosh ^{2} \xi-\sinh ^{2} \xi}{\cosh ^{2} \xi}}=\frac{1}{\cosh \xi} \rightarrow\left(\cosh ^{2} \xi-\sinh ^{2} \xi=1\right)
\end{aligned}
$$

It is totally in accordance with the [5] and with the [6]:

$$
\cosh \xi=\frac{1}{\sqrt{1-\tanh ^{2} \xi}}
$$

\{See Equation (14)\}: $\tanh \xi=(v / c)\}$.

16) See Equation (15):

$$
\sin \varphi=\frac{1}{\cosh \xi}=\sqrt{1-\tanh ^{2} \xi}
$$

17) See Equation (16):

$$
\frac{1}{\sin \varphi}=\cosh \xi\{\text { radian }\}
$$

We got the first new equation. We can see that the $\varphi$ variable and the $\xi$ variable depend of each other.

The $\varphi$ variable determines the moving point position on the circle, while the $\xi$ variable determines the moving point position on the hyperbola. And as we mentioned, the $\varphi$ variable and the $\xi$ variable depend only of the speed of $v$.

We shall later substitute the Equation (17) into the Equations ((1) and (2)). But before it, we shall derive the new equation of ( $\sinh \xi$ ) variable which exist in the Equations ((1) and (2)).

\subsection{The Second New Equation}

In the Equation (14): $\{\cos \varphi=\tanh \xi=(v / c)\}$ allows to find connection between ( $\operatorname{cotan} \varphi)$ and $(\sinh \xi)$.

18) Now comes equation cotan $\varphi$ :

$$
\operatorname{cotan} \varphi=\frac{\cos \varphi}{\sin \varphi}=\frac{\cos \varphi}{\sqrt{1-\cos ^{2} \varphi}}
$$

19) We substitute in the Equation (18), Equation (14): $\cos \varphi=\tanh \xi$ and

$$
\begin{gathered}
\text { Equation (16): } \frac{1}{\cosh \xi}=\sqrt{1-\tanh ^{2} \xi} \text { and } \tanh \xi=(\sinh \xi / \cosh \xi) \\
\operatorname{cotan} \varphi=\frac{\cos \varphi}{\sin \varphi}=\frac{\cos \varphi}{\sqrt{1-\cos ^{2} \varphi}}=\frac{\tanh \xi}{\sqrt{1-\tanh ^{2} \xi}}=\frac{(\sinh \xi / \cosh \xi)}{1 / \cosh \xi}=\sinh \xi
\end{gathered}
$$


20) See Equation (19): $\operatorname{cotan} \varphi=\sinh \xi \quad$ \{radian\}.

So we got our second new equation as well. That means that the $\sinh \xi$ and $\cosh \xi$ variables which appear in the Equations (1) and (2), are expressible by the trigonometric functions of the Equations (17) and (20).

\subsection{The New Equation of $x^{\prime}$}

21) We substitute the Equation (17) and Equation (20) into the Equation (2):

$$
x^{\prime}=x \cosh \xi-c t \sinh \xi=\frac{1}{\sin \varphi} x-c t \operatorname{cotan} \varphi
$$

Note: Equation (8): $\varphi=\arccos (v / c)$

Equation (13): $\xi=\operatorname{artanh}(v / c)$

$$
(-1.0 \leq(v / c) \leq 1.0)
$$

It is one of most important equation of this work. It is distinct that the both sides of equation give the same result. So the hyperbolic Equation (2), we can substitute by a trigonometric equation.

It is very important to prove that the Equations ((17) and (20)) are correct. Further we will prove the correctness of Equations ((17) and (20)) in several different ways.

Now we check the correctness of the Equation (21). So by using the new equations we derive the know equation of Lorentz transformation. So follows the known equation of $x^{\prime}$ :

$$
\operatorname{cotan} \varphi=\frac{\cos \varphi}{\sin \varphi}
$$

22) Equation (21):

$$
x^{\prime}=\frac{1}{\sin \varphi} x-c t \operatorname{cotan} \varphi=\frac{1}{\sin \varphi} x-c t \frac{\cos \varphi}{\sin \varphi}=\frac{x-c t \cos \varphi}{\sin \varphi}
$$

See Equation (9): $\sin \varphi=\sqrt{1-\left(v^{2} / c^{2}\right)}$ and see Equation (7): $\cos \varphi=(v / c)$

23) So:

$$
x^{\prime}=\frac{x-c t \cos \varphi}{\sin \varphi}=\frac{x-c t(v / c)}{\sin \varphi}=\frac{x-v t}{\sqrt{1-\left(v^{2} / c^{2}\right)}}
$$

Form the Equation (21) we got exactly the known equation of Lorentz transformation. So that means that the Equation (21) is correct. Here it is clearly visible that the point of the Equation (21) is the simplicity.

\subsection{The New Equation of $c t^{\prime}$}

For the determination of $c t^{\prime}$ the same procedure is used.

24) Now come the Equation (1): $c t^{\prime}=c t \cosh \xi-x \sinh \xi$

25) We substitute the Equation (17) and Equation (20) into the Equation (1):

$$
c t^{\prime}=c t \cosh \xi-x \sinh \xi=c t \frac{1}{\sin \varphi}-x \operatorname{cotan} \varphi
$$

Now we check the correctness of the Equation (25). By the usage the new Equation (25) it is derived the Lorentz transformation other know equation $t^{\prime}$.

Here we use the same procedure as above by the equation $x^{\prime}$.

$$
\operatorname{cotan} \varphi=(\cos \varphi) /(\sin \varphi)
$$

26) See Equation (25):

$$
c t^{\prime}=c t \frac{1}{\sin \varphi}-x \operatorname{cotan} \varphi=c t \frac{1}{\sin \varphi}-x \frac{\cos \varphi}{\sin \varphi}=\frac{c t-x \cos \varphi}{\sin \varphi}
$$

27) See Equation (7): $\cos \varphi=(v / c)$ 
See Equation (9): $\sin \varphi=\sqrt{1-\left(v^{2} / c^{2}\right)}$

$$
c t^{\prime}=\frac{c t-x \cos \varphi}{\sin \varphi}=\frac{c t-(v / c) x}{\sin \varphi}=\frac{c t-(v / c) x}{\sqrt{1-\left(v^{2} / c^{2}\right)}}=c \frac{t-\left(v / c^{2}\right) x}{\sqrt{1-\left(v^{2} / c^{2}\right)}}
$$

28) So:

$$
t^{\prime}=\frac{t-\left(v / c^{2}\right) x}{\sqrt{1-\left(v^{2} / c^{2}\right)}}
$$

From the Equation (25) we got exactly the known equation of Lorentz transformation. So that means that the Equation (25) is correct. Here it is clearly visible that the point of the Equation (25) is the simplicity.

\subsection{Now We Check the Correctness of the Equation (21) and Equation (25)}

Equation (21):

$$
x^{\prime}=\frac{1}{\sin \varphi} x-c t \operatorname{cotan} \varphi
$$

Equation (25):

$$
c t^{\prime}=c t \frac{1}{\sin \varphi}-x \operatorname{cotan} \varphi
$$

Minkowsi's equation: $x^{\prime 2}-c^{2} t^{\prime 2}=x^{2}-c^{2} t^{2}$

$$
\begin{gathered}
x^{\prime 2}-c^{2} t^{\prime 2}=\left(\frac{1}{\sin \varphi} x-c t \operatorname{cotan} \varphi\right)^{2}-\left(c t \frac{1}{\sin \varphi}-x \operatorname{cotan} \varphi\right)^{2} \\
x^{\prime 2}-c^{2} t^{\prime 2}=\left(\frac{1}{\sin \varphi} x\right)^{2}+(c t \operatorname{cotan} \varphi)^{2}-2 x c t \frac{\operatorname{cotan} \varphi}{\sin \varphi}-\left(\frac{1}{\sin \varphi} c t\right)^{2}-(x \operatorname{cotan} \varphi)^{2}+2 x c t \frac{\operatorname{cotan} \varphi}{\sin \varphi} \\
\operatorname{cotan} \varphi=\frac{\cos \varphi}{\sin \varphi} \\
x^{\prime 2}-c^{2} t^{\prime 2}=x^{2}\left(\frac{1}{(\sin \varphi)^{2}}-\frac{(\cos \varphi)^{2}}{(\sin \varphi)^{2}}\right)-c^{2} t^{2}\left(\frac{1}{(\sin \varphi)^{2}}-\frac{(\cos \varphi)^{2}}{(\sin \varphi)^{2}}\right) \\
1-(\cos \varphi)^{2}=(\sin \varphi)^{2} \\
\text { So }: x^{\prime 2}-c^{2} t^{\prime 2}=x^{2}-c^{2} t^{2}
\end{gathered}
$$

So that means that the Equation (21) and Equation (25) are correct. It is visible that we got simply the wellknown Minkowsi's equation.

\subsection{The Repeated Derivation of the New Equations from Figure 4}

The aim of this derivation is to check the correctness of the Equations ((21) and (25)).

On the Figure 4 the Figure $1 O A D$ triangle is simple drawn again. From this triangle are again derived the Equations ((21) and (25)), but here are used the equations of Lorentz transformation as well. The $c t^{\prime}$ equation won't be derived again only the $x^{\prime}$ equation.

29) This derivation needs the visible equations from the "Appendix I" of the book [1]:

$$
a=\frac{1}{\sqrt{1-\left(v^{2} / c^{2}\right)}} ; \quad b=\frac{(v / c)}{\sqrt{1-\left(v^{2} / c^{2}\right)}}
$$




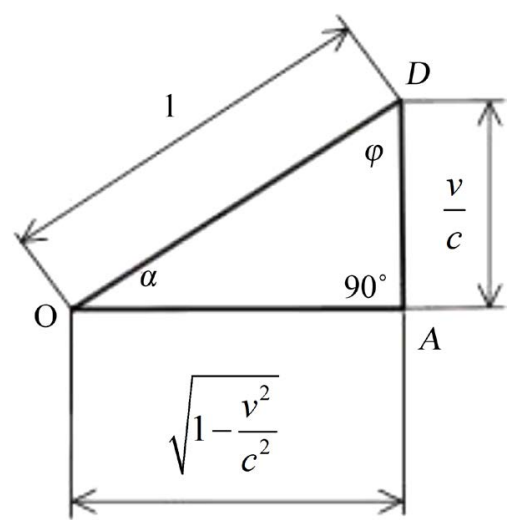

Figure 4. This drawing is used to prove the correctness of Equations ((21) and (25)).

30) See Figure 4 and Equation (29):

$$
\frac{1}{\sin \varphi}=\frac{1}{\sqrt{1-\left(v^{2} / c^{2}\right)}}=a
$$

Equations (17): (1/sin $\varphi=\cosh \xi)$. It is totally in accordance with the [5] and with the [6]:

$$
\cosh \xi=1 / \sqrt{1-\beta^{2}}=\gamma \text { and } \beta=(v / c)
$$

31) See Figure 4, and Equation (7): $\cos \varphi=v / c$

$$
\cos \varphi=\frac{v}{c}=\frac{v t}{c t} \rightarrow v t=c t \cos \varphi
$$

32) See Figure 4 and Equation (29):

$$
\operatorname{cotan} \varphi=\frac{(v / c)}{\sqrt{1-\left(v^{2} / c^{2}\right)}}=b
$$

Equations (20): ( $\operatorname{cotan} \varphi=\sinh \xi)$. It is totally in accordance with the [5] and with the [6]:

$$
(\sinh \xi=(v / c) \gamma) \text {. }
$$

33) We substitute the Equation (30) (1/ $\sin \varphi=a)$, and Equation (32) ( $\operatorname{cotan} \varphi=b)$, and Equation (31) $\left(v t=c t \cos \varphi\right.$ ) into the Equation (29): $x^{\prime}=a x-b c t$.

$$
x^{\prime}=a x-b c t=\frac{1}{\sin \varphi} x-c t \operatorname{cotan} \varphi=\frac{1}{\sin \varphi} x-c t \frac{\cos \varphi}{\sin \varphi}=\frac{x-c t \cos \varphi}{\sin \varphi}=\frac{x-v t}{\sin \varphi}
$$

It is visible that we got the same result with the equation $\left(x^{\prime}=a x-b c t\right)$ by Lorentz transformation, as above with the Equation (22).

So that means that the Equation (21), $\left\{x^{\prime}=x(1 / \sin \varphi)-c t \operatorname{cotan} \varphi\right\}$ is correct.

\section{Second Part}

\subsection{We Introduce First the $\left(t^{\prime} / t\right)$ Formula}

In the references at the end of the work in the "Appendix I" of the book marked by number [1] the Lorentz transformation starts from the fact that it measures in the standing and movable coordinate system the same two oncoming light rays. In the mentioned book, there is given the other derivational way too, when from the standing coordinate system are measured the features of the movable coordinate system. It is written in [1] The behaviour of measuring-rods and clocks in motion chapter of the mentioned book.

In the following derivation we measure the same light-ray from the standing and moving coordinate system. Exclusively in this case can be used that we substitute in the Equation (28) the $x=c t$ equality. The $x=c t$ equal- 
ity substitution in the Equation (28) is given in [1] Lorentz transformation chapter.

So the travelled distance of the light-ray in the standing coordinate system is $x=c t$. Naturally in the movable coordinate system the travelled distance of the light-ray is $x^{\prime}=c t^{\prime}$.

From now on we shall study the travel distance of the light-ray in the standing and movable coordinate system. This gives the opportunity to derive Minkowski's equation. From the view of the special relativity theorem it is very important to derive the Minkowski's equation from the Lorentz transformation

So we take the Equation (28) and pick out the $(t)$ variable. (Note: $(x=c t) ;(x / c)=t$ )

34) See Equation (28):

$$
t^{\prime}=\frac{t-\left(v / c^{2}\right) x}{\sqrt{1-\left(v^{2} / c^{2}\right)}}=\frac{t-(v / c)(x / c)}{\sqrt{1-\left(v^{2} / c^{2}\right)}}=\frac{t-(v / c) t}{\sqrt{1-\left(v^{2} / c^{2}\right)}}=t \frac{1-(v / c)}{\sqrt{1-\left(v^{2} / c^{2}\right)}}
$$

35) Equation $\left(t^{\prime} / t\right)$ :

$$
\frac{t^{\prime}}{t}=\frac{1-(v / c)}{\sqrt{1-\left(v^{2} / c^{2}\right)}}
$$

\subsection{The Third New Equation}

36) The $\tan (\varphi / 2)$ equation can be found in every high school-level mathematic book.

For example, [4]:

$$
\tan (\varphi / 2)=\frac{1-\cos \varphi}{\sin \varphi}=\frac{\sin \varphi}{1+\cos \varphi}=\sqrt{\left(\frac{1-\cos \varphi}{1+\cos \varphi}\right)}\{\operatorname{rad}\}
$$

37) We substitute Equation (7): ( $\cos \varphi=v / c$ ) and Equation (9) into the Equation (36):

$$
\tan (\varphi / 2)=\frac{1-(v / c)}{\sqrt{1-\left(v^{2} / c^{2}\right)}}=\frac{\sqrt{1-\left(v^{2} / c^{2}\right)}}{1+(v / c)}=\sqrt{\left(\frac{1-(v / c)}{1+(v / c)}\right)}\{\operatorname{rad}\}
$$

38) Course, the first member the Equation (37), is identical with Equation (35):

$$
\frac{t^{\prime}}{t}=\frac{c t^{\prime}}{c t}=\tan (\varphi / 2)=\frac{1-(v / c)}{\sqrt{1-\left(v^{2} / c^{2}\right)}}
$$

The Equation (38) can be found in [5].

The third member the Equation (37) can be found in [7].

\subsection{Equations from the "Appendix I" of the Book}

In the introductory part we already mentioned, that it is very important that the equation $\left(t^{\prime} / t\right)=(\tan (\varphi / 2))$ is in accordance with Minkowski's equation. Further on, we are going to deal with proving of it.

In further derivation we shall use the following equations from the Appendix I of the book [1]:

39) The equations:

$$
\begin{gathered}
x^{\prime}=a x-b c t \\
c t^{\prime}=a c t-b x \\
a=\frac{1}{\sqrt{1-\left(v^{2} / c^{2}\right)}} ; \quad b=\frac{(v / c)}{\sqrt{1-\left(v^{2} / c^{2}\right)}} \\
a=\frac{\lambda+\mu}{2} ; \quad b=\frac{\lambda-\mu}{2} \\
\left(x^{\prime}+c t^{\prime}\right)=\mu(x+c t) ; \quad\left(x^{\prime}-c t^{\prime}\right)=\lambda(x-c t)
\end{gathered}
$$


40) From the last equations we express the $\mu$ and $\lambda$ variables:

$$
\mu=\frac{x^{\prime}+c t^{\prime}}{(x+c t)} ; \quad \lambda=\frac{x^{\prime}-c t^{\prime}}{(x-c t)}
$$

First of all, we calculate again the " $a$ " and " $b$ ” variables of the Lorentz transformation from $\tan (\varphi / 2)$ formula.

Then by our " $a$ " and " $b$ " equations we derive the $\mu$ and $\lambda$ variables. We will prove that $\mu=1 / \lambda$ and then by usage of Equation (40) we shall easily get Minkowski’s equation.

\subsection{The Derivation of the Variable " $a$ " and " $b$ "}

Now we continue the Equation (38).

Now we transform the Equation (38) so that we can determine the Lorentz transformation " $a$ " and " $b$ ” variables.

41) We shall transform the Equation (38) and take the Equation (39) into account:

$$
\frac{t^{\prime}}{t}=\tan (\varphi / 2)=\frac{1-(v / c)}{\sqrt{1-\left(v^{2} / c^{2}\right)}}=\frac{1}{\sqrt{1-\left(v^{2} / c^{2}\right)}}-\frac{(v / c)}{\sqrt{1-\left(v^{2} / c^{2}\right)}}=a-b
$$

42) Taking in account the Equations ((41), and (30), (32)):

Equation (30): $a=(1 / \sin \varphi)=\cosh \xi$

Equation (32): $\quad b=\operatorname{cotan} \varphi=\sinh \xi$

$$
\frac{t^{\prime}}{t}=\frac{c t^{\prime}}{c t}=\tan (\varphi / 2)=a-b=\frac{1}{\sin \varphi}-\operatorname{cotan} \varphi=\cosh \xi-\sinh \xi=\mathrm{e}^{-\xi}
$$

Note:

Equation (8): $\varphi=\arccos (v / c)$

Equation (13): $\xi=\operatorname{artanh}(v / c)$

$$
(-1.0 \leq(v / c) \leq 1.0)
$$

By this we proved that the $\tan (\varphi / 2)$ and the hyperbolic equations are connected with each other.

We mustn't forget that we started from the fact that we measure from the standing and movable coordinate system the two oncoming light-rays. In the standing coordinate system, the travelled distance of the light-ray is $x$ $=c t$. From here we started, we substituted it in the Equation (28) and could not get other result. If we look closer the Equation (25) and substitute the equation $x=c t$, it gives the same result.

At the Equation (41) we wrote the $\tan (\varphi / 2)$ equation. Now we write the $\operatorname{cotan}(\varphi / 2)$ mathematical equation too. And here we take into account the equations of (39). The equation got in this way, we shall later use for derivation of variables " $\mu$ ” and “ $\lambda$ ” in Lorentz transformation.

The second member of the Equation (37):

$$
\tan (\varphi / 2)=\frac{\sqrt{1-\left(v^{2} / c^{2}\right)}}{1+(v / c)}
$$

43) So:

$$
\operatorname{cotan}(\varphi / 2)=\frac{1}{\tan (\varphi / 2)}=\frac{1+(v / c)}{\sqrt{1-\left(v^{2} / c^{2}\right)}}=\frac{1}{\sqrt{1-\left(v^{2} / c^{2}\right)}}+\frac{(v / c)}{\sqrt{1-\left(v^{2} / c^{2}\right)}}=a+b
$$

\subsection{The Derivation of the Variable}

The following derivation aim is to prove that: $(\mu=1 / \lambda)$

Equation (39):

$$
a=\frac{\lambda+\mu}{2} ; \quad b=\frac{\lambda-\mu}{2}
$$


We express the $\mu$ and $\lambda$ variables:

44) Variable $\lambda$ :

$$
a=\frac{\lambda+\mu}{2} \rightarrow \lambda=2 a-\mu
$$

45) Variable $\lambda$ :

$$
b=\frac{\lambda-\mu}{2} \rightarrow \lambda=2 b+\mu
$$

46) See Equations ((44) and (45)): $\mu=a-b$

$$
\text { Equation (41): } \tan (\varphi / 2)=a-b
$$

47) See Equation ((46) and (41)):

$$
\mu=a-b=\tan (\varphi / 2)
$$

48) We substitute Equation (46) ( $\mu=a-b$ ) variable, in the Equation (44): $\lambda=2 a-\mu$.

$$
\lambda=2 a-(\mu)=2 a-(a-b)=a+b=\operatorname{cotan}(\varphi / 2)
$$

Equation (43): $\operatorname{cotan}(\varphi / 2)=a+b$

49) From the Equations ((47) and (48)), ( $\mu=(1 / \lambda))$ :

$$
\tan (\varphi / 2)=\frac{1}{\operatorname{cotan}(\varphi / 2)} \rightarrow \mu=\frac{1}{\lambda}
$$

So we calculated “ $\mu$ ” and “ $\lambda$ ”.

\subsection{The Derivation of the Minkowski Equation}

The equations of " $\mu$ ” and " $\lambda$ ” can be found at the Equation (40):

$$
\mu=\frac{x^{\prime}+c t^{\prime}}{(x+c t)} ; \quad \lambda=\frac{x^{\prime}-c t^{\prime}}{(x-c t)}
$$

See equations of (49) and equations of (40):

$$
\begin{gathered}
\mu=\frac{1}{\lambda} \\
\mu=\frac{x^{\prime}+c t^{\prime}}{(x+c t)}=\frac{1}{\left(x^{\prime}-c t^{\prime}\right) /(x-c t)}=\frac{1}{\lambda} \\
\left(x^{\prime}+c t^{\prime}\right)\left(x^{\prime}-c t^{\prime}\right)=(x+c t)(x-c t) \\
x^{\prime 2}-c^{2} t^{\prime 2}=x^{2}-c^{2} t^{2}
\end{gathered}
$$

With this we derived Minkowsi's equation. We proved that Minkowsi's equation is the integral part of the Lorentz transformation. With that, we proved the legitimacy of $\tan (\varphi / 2)$ equation.

If Minkowski's equation is expanded by coordinates " $y$ " and " $z$ ", then we get the four dimensional word logical equation [1]:

$$
z^{\prime 2}+y^{\prime 2}+x^{\prime 2}-c^{2} t^{\prime 2}=z^{2}+y^{2}+x^{2}-c^{2} t^{2}
$$

The reason why the equation can be expanded by " $y$ " and " $z$ " coordinates comes from the fact that the derivation of the Lorentz transformation starts from that simplification that all actions happen on the " $x$ " axe.

\section{Results}

Equation (8): $\varphi=\arccos (v / c)$

Equation (13): $\xi=\operatorname{artanh}(v / c)$ 


$$
(-1.0 \leq(v / c) \leq 1.0)
$$

The equations $c t$ :

Equations (25)-(28):

$$
c t^{\prime}=c t \frac{1}{\sin \varphi}-x \operatorname{cotan} \varphi=c \frac{t-\left(v / c^{2}\right) x}{\sqrt{1-\left(v^{2} / c^{2}\right)}}=c t \cosh \xi-x \sinh \xi
$$

Equations (42):

$$
\begin{gathered}
\text { \{Condition: } x=c t \text { \} } \\
\frac{t^{\prime}}{t}=\tan (\varphi / 2)=a-b=\frac{1}{\sin \varphi}-\operatorname{cotan} \varphi=\cosh \xi-\sinh \xi=\mathrm{e}^{-\xi}
\end{gathered}
$$

The $x^{\prime}$ equations:

Equation (21):

$$
x^{\prime}=\frac{1}{\sin \varphi} x-c t \operatorname{cotan} \varphi=x \cosh \xi-c t \sinh \xi
$$

\section{Conclusions}

We multiply all sides of the triangle on the Figure $4 O A D$ by " $c t$ ". The triangle got in this way is the $O A D$ triangle on the Figure 5.

The $O A$ distance on the Figure 5 is:

$$
\sqrt{(c t)^{2}-(v t)^{2}}=\sqrt{(c t)^{2}\left(1-\frac{v^{2}}{c^{2}}\right)}=c t \sqrt{\left(1-\frac{v^{2}}{c^{2}}\right)}
$$

By this we showed that on the Figure 5, the triangle "OA" side is determined by Pythagoras theorem, and the Pythagoras theorem is the geometrical mean of the $(c t-v t)$ and $(c t+v t)$ numbers:

$$
\sqrt{(c t-v t)(c t+v t)}=\sqrt{(c t)^{2}-(v t)^{2}} \text {. }
$$

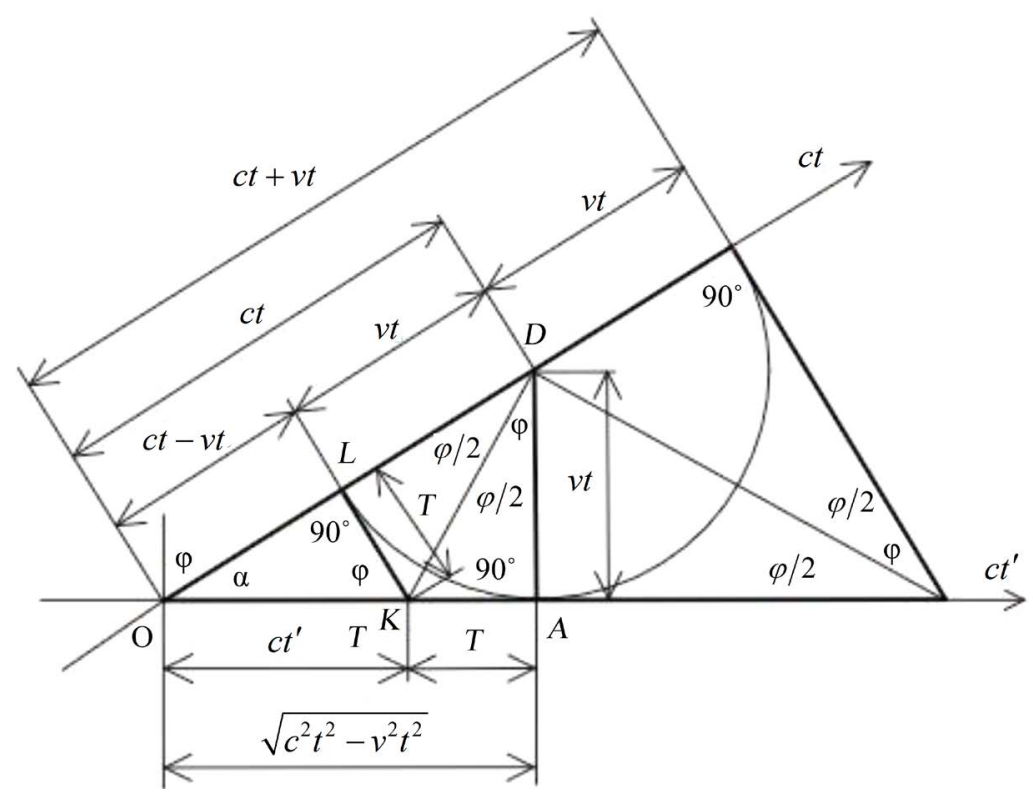

Figure 5. If the $A D=v t=0.0$, then $c t$ and $c t^{\prime}$ coordinate the axe line up. If we change the value of $v t$, then it will change the value of $\varphi$ and $\alpha$ as well. 
We can draw the $(c t+v t)$ dimension and $\tan (\varphi / 2)$ on the Figure 5 .

See Figure 5, triangle $O A D$ and triangle $O K L$ :

$$
\begin{gathered}
\sin \varphi=\frac{\sqrt{(c t)^{2}-(v t)^{2}}}{c t}=\frac{c t-v t}{c t^{\prime}} \\
\text { So }: \frac{c t^{\prime}}{c t}=\frac{t^{\prime}}{t}=\frac{c t-v t}{\sqrt{(c t)^{2}-(v t)^{2}}}=\frac{t(c-v)}{t \sqrt{(c)^{2}-(v)^{2}}}=\frac{c-v}{\sqrt{\left(c^{2}-v^{2}\right)}}=\frac{1-(v / c)}{\sqrt{1-\left(v^{2} / c^{2}\right)}} .
\end{gathered}
$$

Figure 5 and last equation shows the Equation (37) and Equation (38).

Equation (37):

$$
\frac{t^{\prime}}{t}=\tan (\varphi / 2)=\frac{1-(v / c)}{\sqrt{1-\left(v^{2} / c^{2}\right)}}=\frac{\sqrt{1-\left(v^{2} / c^{2}\right)}}{1+(v / c)} .
$$

Proof:

See Figure 5, triangle $O K L$ and $O A D$ :

$$
\begin{gathered}
\cos \varphi=\frac{T}{c t^{\prime}}=\frac{v t}{c t} \\
\text { So }: \frac{T}{v t}=\frac{c t^{\prime}}{c t}
\end{gathered}
$$

See Figure 5, triangle $K L D$, and $K A D$ :

$$
\begin{gathered}
\tan (\varphi / 2)=\frac{T}{v t} \\
\text { So : } \tan (\varphi / 2)=\frac{T}{v t}=\frac{c t^{\prime}}{c t}=\frac{t^{\prime}}{t}
\end{gathered}
$$

So, that means that the Figure 5 shows the Equation (37) and Equation (38). The (ct') coordinate axe is rotated by $(90-\varphi)=\alpha$ angle comparing to the $(c t)$ coordinate axe, so that $(\cos \varphi=v / c)$.

\section{Discussion}

Watching from the great speed moving coordinate system, the world seems to be deformed, while watching from the standing coordinate system the same world looks undeformed. But the same world cannot be deformed and undeformed at the same time. The higher the speed of a moving coordinate system, the more it seems to be deformed the world from there. This phenomenon is closer to the Doppler effect. So, if on the Figure 5, the $c t^{\prime}$ rotation is caused by a force field, then the Figure 5 is right. But if on the Figure 5 there is no force field, then we are dealing whit a Doppler effect. Here it comes that in accordance with the general theory of relativity, the gravitational force field phenomenon can be changed with an accelerated, but without force field coordinate system.

Figure 3: Our world is ball.

\section{References}

[1] Einstein, A. (1921) Relativity: The Special and the General Theory. http://www.gutenberg.lib.md.us/3/6/1/1/36114/36114-pdf.pdf

[2] Nima, A.B. (2013) Remarks around Lorentz Transformation. https://hal.inria.fr/file/index/docid/875708/filename/Remarks around Lorentz Transformation.pdf

[3] Wikipedia. https://en.wikipedia.org/wiki/Lorentz_transformation

[4] Bronstejn, I.N. and Szemengyajev, K.A. (1982) Matematikai Zsebkönyv. Translate: Mathematical Pocketbook.

[5] Rindler, W. (2006) Relativity: Special, General, and Cosmological. http://dx.doi.org/10.1119/1.1622355

[6] Ryder, L.H. (1996) Quantum Field Theory. [Online]. http://dx.doi.org/10.1017/CBO9780511813900

[7] Popescu, B.R.A.S. Lorentz Transformation, Time Dilation, Length Contraction and Doppler Effect-All at Once. http://arxiv.org/pdf/0712.0463 